\title{
Prolonged Fetal Heart Rate Decelerations in Labor: Can We Reduce Unplanned Primary Cesarean Sections in This Group?
}

\author{
John A. Morgan · Miriam E. Hankins · Yuping Wang · \\ Donna Hutchinson - Hannah L. Sams - John H. Voltz • \\ Charles E. McCathran · Alan D. Kaye $\cdot$ David F. Lewis
}

Received: June 22, 2020 / Published online: August 24, 2020

(C) The Author(s) 2020

\begin{abstract}
Introduction: Non-reassuring fetal tracing is the second leading cause of primary cesarean delivery in the United States. Prolonged fetal heart rate decelerations are non-reassuring fetal heart rate characteristics, which do not uniformly predict poor fetal outcome but can prompt obstetricians to proceed with cesarean delivery. The objective of this manuscript is to identify a strategy to reduce the primary cesarean section rate in patients with prolonged fetal heart rate decelerations in labor.

Methods: This is a retrospective cohort study over a 5-year period at an academic medical center, including patients undergoing primary cesarean section following labor induction, augmentation, or spontaneous labor who were noted to have prolonged fetal heart rate deceleration(s) in the $1 \mathrm{~h}$ prior to the time of delivery. Two groups were compared: "crash" cesarean sections versus "emergent" cesarean sections. The primary outcome was if fetal heart
\end{abstract}

Digital Features To view digital features for this article go to https://doi.org/10.6084/m9.figshare.12777242.

J. A. Morgan · M. E. Hankins · Y. Wang ·

D. Hutchinson - H. L. Sams · J. H. Voltz .

C. E. McCathran - A. D. Kaye $(\bowtie) \cdot$ D. F. Lewis

Department of Obstetrics and Gynecology,

Louisiana State University Health Sciences Center,

Shreveport, Shreveport, LA, USA

e-mail: akaye@lsuhsc.edu tones were rechecked in the operating room prior to cesarean section incision. Secondary outcomes included maternal-fetal monitoring versus Doppler fetal heart tones in the operating room, return to baseline noted in the operating room, fetal outcomes, fetal monitoring characteristics, and anesthesia type between crash versus emergent groups.

Results: Of 1969 term singleton cesarean sections, 119 patients met our inclusion criteria (emergent group $n=80$ ) (crash group $n=39$ ), which accounted for $13.9 \%$ of all primary cesarean sections during the study period. The emergent group had a significantly higher rate of reassessment of fetal heart tones in the operating room $n=61(76.2 \%)$ versus the crash group $n=15(38.4 \%)(p \leq 0.0001)$. There were no statistically significant differences regarding fetal outcomes between the two groups. The crash group had a higher rate of category 1 fetal heart rate tracing prior to the prolonged deceleration, a longer median prolonged deceleration, and a deeper median nadir of the prolonged deceleration; these differences were statistically significant. The prolonged-to-delivery interval was significantly shorter in the crash group (median $=15 \mathrm{~min}$ ) than tin he emergent group (median $=33 \mathrm{~min}) \quad(p$ $\leq 0.0001)$. The crash group also had a higher rate of general anesthesia $(n=11,28.2 \%)$ than the emergent group $(n=6,7.5 \%)(p=0.002)$. The crash group was specifically investigated. Of the 15 patients with fetal heart tones rechecked 
in the crash group, 7 had returned to baseline in the operating room, but underwent cesarean section without fetal monitoring.

Conclusion: Our results indicate that the practice of placing patients on fetal monitor upon arrival to the operating room prior to performing crash cesarean delivery could reduce the rate of primary cesarean deliveries performed for prolonged decelerations in labor. When fetal heart tones have returned to baseline upon arrival in the operating room, the decision to proceed with cesarean delivery can be reconsidered. However, many clinical factors must be taken into consideration, and the decision to proceed is ultimately at the discretion of the obstetrics provider.

Keywords: Crash; Deceleration; Electronic fetal monitoring; Emergent; Labor; Primary cesarean section; Prolonged

\section{Key Summary Points}

The practice of placing patients on fetal monitor upon arrival to the operating room prior to performing crash cesarean delivery could reduce a significant number of primary cesarean deliveries with prolonged decelerations in labor.

Additional time can allow for intrauterine resuscitation and the delivery of regional anesthesia techniques which afford additional benefits to the patient and newborn.

Additional time can provide for resolution of the insult.

If fetal heart tones have returned to baseline upon arrival to the operating room, the decision to proceed with cesarean delivery can be reconsidered.

The clinical decision to proceed with cesarean delivery must be made taking into account many clinical factors and is the decision of the obstetrics provider.

\section{INTRODUCTION}

Over the last two decades, there has been a nationwide increase in the United States in the cesarean delivery rate, from $20.7 \%$ in 1996 to $32.7 \%$ in 2013 [1-3]. The increase in the cesarean delivery rate has not been associated with a concomitant decrease in maternal and neonatal morbidity or mortality, which has led experts to believe that cesarean delivery is being over-utilized [1]. Attention was turned to reducing the primary cesarean delivery rate to ultimately decrease overall cesarean delivery rate. The two leading causes of primary cesarean delivery in the United States are labor arrest (34\%) and non-reassuring fetal status (23\%) [1].

Electronic fetal monitoring (EFM) is used in $85 \%$ of all laboring patients in the United States, making EFM the most common obstetrics procedure [4-6]. When compared with intermittent auscultation, EFM is associated with increased operative delivery without evidence of decreased fetal or newborn morbidity or mortality [4-7].

Prolonged fetal heart rate deceleration, defined as a visually apparent decrease in the fetal heart rate below baseline by $15 \mathrm{bpm}$ for longer than $2 \mathrm{~min}$ but less than $10 \mathrm{~min}$, is an example of a non-reassuring fetal heart rate characteristic, which often occurs in response to physiologic and reversible changes to maternal or fetal status [8]. Some examples of causes include rapid cervical change, uterine tachysystole, cervical examination, maternal hypotension from regional anesthesia placement, and artificial rupture of membranes [8].

When prolonged decelerations occur in labor, they are stressful events for both the patients and the labor and delivery staff. The presence of a prolonged deceleration can lead to emergency cesarean delivery if resolution does not occur promptly. In unplanned primary cesarean delivery, fetal monitoring should continue until abdominal sterile preparation has begun. If internal monitoring with a fetal scalp electrode is used, fetal monitoring should continue until abdominal sterile preparation is completed [9]. The objective of the present study, therefore, is to investigate the rate of EFM 
in the operating room in patients delivered with prolonged fetal heart rate decelerations in labor, in order to identify a strategy to reduce the primary cesarean delivery rate in this cohort.

\section{METHODS}

\section{Study Population}

A retrospective cohort study was performed by the Department of Obstetrics and Gynecology, University Health Hospital in Shreveport, Louisiana, in affiliation with Louisiana State University Health Sciences Center-Shreveport (LSUHSC-Shreveport). Patients were included over a 5-year period ending in January, 2017. All patients delivered by cesarean section were identified and stratified by indication. Inclusion criteria for evaluation included term, singleton gestations delivered by primary cesarean delivery for non-reassuring fetal tracing. An EFM certified physician evaluated the EFM in the 1-h prior to delivery for each patient who met our inclusion criteria. Patients were excluded if EFM was insufficient for evaluation, in the absence of a prolonged deceleration, if placental abruption was noted at the time of delivery, or in the presence of umbilical cord prolapse. Patients with umbilical cord prolapse and placental abruption were excluded because primary cesarean delivery was considered unavoidable in this group. There were no cases of uterine rupture in the study population.

The study population was divided into two groups: "crash" cesarean sections and "emergent" cesarean sections. A cesarean section would be included into either the "crash" or "extremely urgent" group, if so specified in the medical record or if there was immediate need for delivery related to concerns for maternal or fetal well-being [10]. The "emergent" group included all other patients delivered for nonreassuring fetal status with prolonged decelerations in labor, which were not "extremely urgent" requiring immediate delivery, e.g., "crash" cesarean sections. A maximum 1-h prolonged-to-delivery interval (defined as time from the onset of the prolonged deceleration to delivery of the fetus) was chosen as a cut-off for inclusion. It is documented in the literature that even a well-staffed, Level III hospital, the ability to achieve the ACOG standard of less than 30 min from decision to delivery is challenging $[11,12]$. The percentage of patients for whom this goal was met is beyond the scope of the present study. We chose $1 \mathrm{~h}$ as a cutoff for inclusion to ensure no patients delivered for non-reassuring fetal tracing were incidentally excluded from the study.

Maternal and neonatal information was obtained through linked charts in EPIC electronic medical record system. EFM strips were obtained using the OBIX Perinatal Data System. The Institutional Review Board at LSUHSCShreveport approved our study.

\section{Outcomes}

The primary study outcome was whether fetal heart tones were rechecked in the operating room prior to incision for cesarean section. Multiple secondary outcomes were also investigated. Reassessment of fetal heart tones was separated into EFM versus measurement of fetal heart tones with an external Doppler or fetal scalp electrode at a single point in time. The percentage of the "crash" group who had recovered on arrival to the operating room was also noted. Another secondary outcome investigated was neonatal outcomes. Outcomes compared between the two groups included $\mathrm{pH}$ less than 7.2, $\mathrm{pH}<7.1,5$-min APGAR score $<7$, need for neonatal resuscitation, and admission to the neonatal intensive care unit. During the study period, umbilical cord gases were not routinely collected on all patients delivered for non-reassuring fetal status, especially in the presence of a vigorous newborn. Cord gases were only included in the study if they were specified to be arterial in the laboratory report. Unspecified cord gases or venous-only cord gases were excluded from the study. Neonatal resuscitation techniques included are as follows: positive pressure ventilation, bubble continuous positive airway pressure, and bag mask ventilation.

The fetal heart rate tracing category prior to the prolonged deceleration for each delivery 
was noted. Category I was defined as the baseline fetal heart rate between 110 and 160 beats per min, moderate beat-to-beat variability, absent late or variable decelerations, with the presence or absence of accelerations $[5,6]$. Category III was defined as absent beat-to-beat variability with recurrent late decelerations, recurrent variable decelerations, bradycardia, or a sinusoidal pattern $[5,6]$. The Category II fetal heart rate pattern includes all EFM tracings not categorized as Category I or III. These tracings are indeterminate and are not predictive of fetal outcome $[5,6,8]$. Prolonged deceleration characteristics, including the length of the prolonged deceleration closest to delivery, the prolonged-to-delivery interval, the nadir of the prolonged deceleration, and the presence or absence of recovery from prolonged deceleration, were documented. Anesthesia type was also noted, including epidural, general, or spinal anesthesia.

\section{Statistical Analysis}

Statistical analysis was performed using computer software GraphPad Prism 7 (La Jolla, CA, USA). All numeric or quantitative data was compared using the unpaired Student's $t$ test. All categorical variables were compared using the Chi square test. A probability level of less than 0.05 was set as statistically significant.

\section{RESULTS}

For the 5-year study period, we identified 1969 term, singleton cesarean sections. After the exclusions of all repeat cesarean sections and primary cesarean sections for indications other than "non-reassuring fetal tracing", 357 patients were eligible for the study. An EFM-certified physician evaluated the monitoring of each of these patients. A total of 119 patients met our inclusion criteria, with 39 in the crash group and 80 in the emergent group (Fig. 1).

The demographics between the crash and emergent groups were compared, including maternal age, BMI, nulliparity, gestational age at delivery, ethnicity (e.g., African American, Caucasian, Hispanic, Asian), past medical history (diabetes, hypertensive disorders, asthma, thyroid disease), augmentation/induction method (misoprostol, oxytocin, Foley bulb) with no significant differences. The crash group had a higher rate of general anesthesia for cesarean section when compared to the emergent group, $n=10(27.7 \%)$ versus $n=7(8.4 \%)$ $(p=0.002)$. The emergent group has a significantly higher rate of epidural anesthesia 71 $(88.7 \%)$ versus $28(71.7 \%)$ (Table 1$)$.

Fetal heart tones were rechecked significantly more often in the emergent group, 61 patients $(76.2 \%)$, versus the crash group, 15 $(38.5 \%) \quad(p<0.0001)$. More patients in the emergent group were monitored with EFM in the operating room than in the crash group. The emergent group had a higher rate of patients at baseline, but nearly $50 \%$ of patients in the crash group who had fetal heart tones rechecked in the operating room had returned to baseline on reassessment (Table 2).

No differences with regards to neonatal outcomes (5-min APGAR less than 7, need for neonatal resuscitation, umbilical artery $\mathrm{pH}$ less than 7.2 or 7.1, or NICU admission) were noted between the two groups (Table 3). Umbilical artery $\mathrm{pH}$ was available in 47 of the 119 patients. Among them, cord gases were tested in 30 of $83(36 \%)$ patients in the emergent group and 17 of $36(47 \%)$ patients in the crash group. Cord $\mathrm{pH}$ less than 7.2 and less than 7.1 were used as categorical variables with regards to statistical analysis.

Fetal heart rate monitoring characteristics were analyzed. The crash cesarean section group had a longer median prolonged fetal heart rate deceleration closest to delivery, $6 \mathrm{~min}$ versus 4 min for the emergent group $(p<0.0001)$, a shorter interval from prolonged deceleration to delivery, $15 \mathrm{~min}$ versus $33 \mathrm{~min}(p \leq 0.0001)$, and a deeper nadir of the prolonged deceleration, 60 beats per min versus 70 beats per min $(p=0.0002)$ when compared to the emergent group. The crash group had a higher rate of category 1 (or reassuring) fetal heart rate prior to the prolonged deceleration $n=12(33.3 \%)$ versus $n=10(12.0 \%) \quad(p=0.003)$ when compared to the emergent group. In contrast, the emergent group had a higher rate of category 2 (indeterminate) fetal heart rate $n=68(81.9 \%)$ 


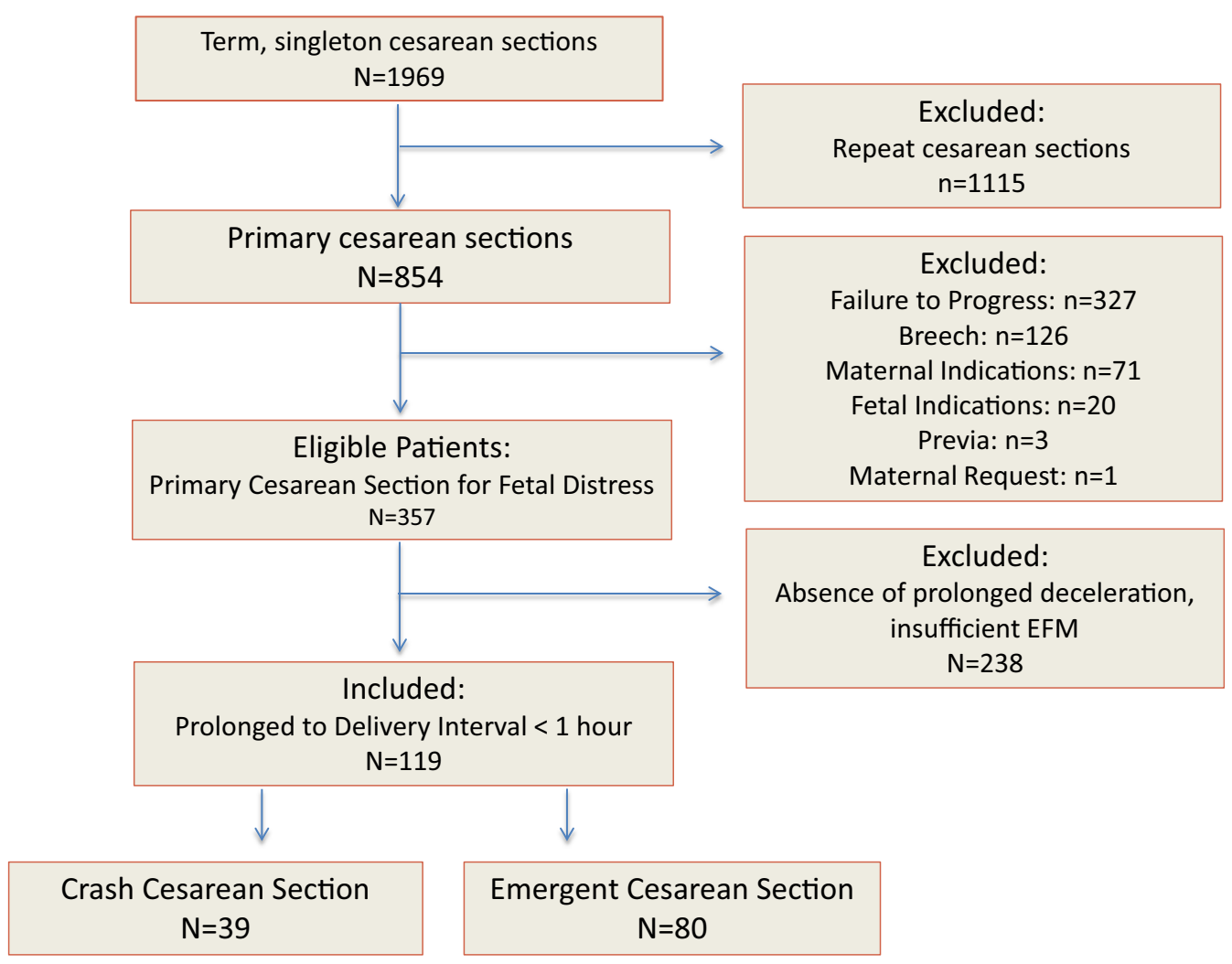

Fig. 1 Flow diagram of patient selection with exclusions

versus $n=23(63.8 \%)(p=0.02)$ when compared to the crash group (Table 4). All data used in the present study were collected in accordance with the Helsinki Declaration.

\section{DISCUSSION}

Prolonged fetal heart rate decelerations frequently occur during labor as isolated events with physiologic and reversible causes [8]. In a significant number of cases, it is common practice to perform a cesarean section in response to fetal heart rate decelerations. Previous studies have documented the lack of association between fetal bradycardia and poor neonatal outcomes [13-16]. Williams et al. [13] reviewed 186 patients in labor with fetal bradycardia. They compared the presence or absence of baseline variability and recovery from the bradycardia with cord $\mathrm{pH}$ at birth. The majority of patients in their study had moderate baseline variability, recovered from the bradycardia, and had satisfactory cord $\mathrm{pH}$ levels. They concluded that delivery following fetal bradycardia often reveals an uncompromized infant, and operative intervention in all of these patients will result in an increase in maternal trauma [13]. The results of the present investigation clearly demonstrated that the practice of placing patients on fetal monitoring upon arrival in the operating room identified a significant number of patients who had fetal heart rate return to baseline in both the crash (46.6\%) and emergent (96.7\%) groups (Table 2).

Our study differs from these previous studies conducted on prolonged fetal heart rate decelerations or fetal bradycardia. Previous studies have investigated only prolonged decelerations in the second stage of labor and included patients delivered vaginally $[15,16]$. Our study investigates prolonged decelerations in all stages of labor, and only includes patients delivered via primary cesarean section. Our study also differs from previous studies as we used a modifiable practice pattern as a primary 
Table 1 Demographics

\begin{tabular}{|c|c|c|c|}
\hline & Crash $(n=39)$ & Emergent $(n=80)$ & $P$ \\
\hline Maternal age (years) & $23(20-26)$ & $25(20-31)$ & 0.161 \\
\hline BMI $\left(\mathrm{kg} / \mathrm{m}^{2}\right)$ & $31.8(27.6-37.6)$ & $34.4(28.4-40.5)$ & 0.494 \\
\hline Nulliparity & $15(38.4)$ & $40(50)$ & 0.236 \\
\hline Gestational age (weeks/days) & $39 w 2 d(38 w 3 d-40 w 0 d)$ & $39 w 2 d(38 w 2 d-40 w 1 d)$ & 0.803 \\
\hline \multicolumn{4}{|l|}{ Ethnicity } \\
\hline African American & $33(84.6)$ & $65(81.3)$ & 0.651 \\
\hline Caucasian & $3(7.6)$ & $10(12.5)$ & 0.43 \\
\hline Hispanic & $2(5.1)$ & $5(6.2)$ & 0.807 \\
\hline Asian & $1(2.5)$ & $0(0)$ & 0.15 \\
\hline Past medical history & $12(30.7)$ & $24(30)$ & 0.931 \\
\hline \multicolumn{4}{|c|}{ Augmentation/induction method } \\
\hline Cytotec & $5(12.8)$ & $11(13.7)$ & 0.889 \\
\hline Pitocin & $25(64.1)$ & $55(68.7)$ & 0.612 \\
\hline Spontaneous labor & $8(20.5)$ & $13(16.2)$ & 0.566 \\
\hline Foley balloon & $0(0)$ & $1(1.2)$ & 0.483 \\
\hline \multicolumn{4}{|l|}{ Anesthesia } \\
\hline Epidural & $28(71.7)$ & $71(88.7)$ & 0.02 \\
\hline General & $11(28.2)$ & $6(7.5)$ & 0.002 \\
\hline Spinal & $0(0)$ & $3(3.7)$ & 0.22 \\
\hline
\end{tabular}

Comparison of maternal demographic characteristics between crash and emergent groups

Data are $n(\%)$ or median (interquartile range)

Table 2 Primary outcome

\begin{tabular}{lllc}
\hline & Crash $(\boldsymbol{n}=\mathbf{3 9 )}$ & Emergent $(\boldsymbol{n}=\mathbf{8 0})$ & $\boldsymbol{P}$ \\
\hline Fetal heart tones rechecked in operating room & $15(38.4)$ & $61(76.2)$ & $<0.0001$ \\
Monitored on EFM in operating room $^{\mathrm{a}}$ & $2(13.3)$ & $39(63.9)$ & $<0.0001$ \\
Checked with ext Doppler $^{\mathrm{a}}$ & $9(60)$ & $15(38.4)$ & 0.008 \\
Checked with FSE $^{\mathrm{a}}$ & $4(26.6)$ & $7(11.4)$ & 0.134 \\
At baseline in operating room $^{\mathrm{a}}$ & $7(46.6)$ & $59(96.7)$ & $<0.0001$ \\
\hline
\end{tabular}

Results including primary outcome with regards to assessment of fetal heart tones on arrival in the operating room Data are $n(\%)$

EFM electronic fetal monitoring, Ext external, FSE fetal scalp electrode

a $\%$ only includes those patients with fetal heart tones rechecked in operating room 
Table 3 Fetal outcomes

\begin{tabular}{lccc}
\hline & Crash $(\boldsymbol{n}=39)$ & Emergent $(\boldsymbol{n}=\mathbf{8 0})$ & $\boldsymbol{P}$ \\
\hline 5 min APGAR $<7$ & $1(2.5)$ & $1(1.2)$ & 0.6 \\
Resuscitation & $4(10)$ & $6(7.5)$ & 0.61 \\
Umbilical artery pH $<7.1^{\mathrm{a}, \mathrm{b}, \mathrm{c}}$ & $1(5.8)$ & $2(6.6)$ & 0.915 \\
Umbilical artery pH $<7.2^{\mathrm{a}, \mathrm{b}, \mathrm{c}}$ & $7(41.1)$ & $7(23.3)$ & 0.198 \\
NICU admission & $7(17.9)$ & $10(12.5)$ & 0.425 \\
Fetal gender & $21(53.8)$ & & 0.523 \\
Male & $18(46.2)$ & $48(60)$ & 0.523 \\
Female & $3100(2785-3400)$ & $32(40)$ & 0.367 \\
Fetal weight $(\mathrm{g})$ & $3062(2688-3348)$ & \\
\hline
\end{tabular}

Comparison of fetal outcomes between the crash and emergent groups

Data are $n(\%)$ or median (interquartile range)

${ }^{a}$ Umbilical cord gases collected in 17 of 39 (43.6\%) of crash group and 30 of 80 (37.5\%) of emergent group

b Crash group 7 of 17 (41.1\%) with umbilical artery pH less than 7.2; 1 of 17 (5.8\%) with umbilical artery pH less than 7.1

c Emergent group 7 of $30(23.3 \%)$ with umbilical artery pH less than $7.2 ; 2$ of $30(6.6 \%)$ with umbilical artery pH less than 7.1

Table 4 Electronic fetal monitoring characteristics

\begin{tabular}{|c|c|c|c|}
\hline & Crash $(n=39)$ & Emergent $(n=80)$ & $\boldsymbol{P}$ \\
\hline \multicolumn{4}{|l|}{ EFM tracing category prior to prolonged deceleration } \\
\hline Category 1 & $13(33.3)$ & $9(11.3)$ & 0.003 \\
\hline Category 2 & $25(64.1)$ & $66(82.5)$ & 0.02 \\
\hline Category 3 & $1(2.5)$ & $5(6.25)$ & 0.388 \\
\hline Length of prolonged deceleration closest to delivery ( $\mathrm{min}$ ) & $6(5-8)$ & $4(3-5)$ & $<0.0001$ \\
\hline Prolonged deceleration to delivery interval ( $\mathrm{min}$ ) & $15(13-17)$ & $33(28.2-45)$ & $<0.0001$ \\
\hline Nadir of prolonged decel (bpm) & $60(50-70)$ & $70(60-80)$ & 0.0002 \\
\hline
\end{tabular}

Comparison of electronic fetal monitoring characteristics between the crash and emergent groups

Data are $n(\%)$ or median (interquartile range)

Category definitions are according to the Eunice Kennedy Shriver National Institute of Child Health and Human Development Three-Tiered Classification of Fetal Heart Rate Tracings [3, 4]

bpm beats per min

outcome in an attempt to identify a strategy to reduce the primary cesarean section rate. While we include fetal outcomes in our analysis, previous studies have investigated neonatal outcomes in the presence of prolonged decelerations as the primary outcome [13-16].
Experts suggest a calm initial clinical response to prolonged fetal heart rate decelerations, to allow time for resolution of the fetal heart rate deceleration, and to prevent unnecessary stress on the patient [8]. The most important initial step when responding to a 
prolonged fetal heart rate deceleration is to rule out catastrophic/irreversible causes, such as uterine rupture, placental abruption, or umbilical cord prolapse. Once these devastating causes are ruled out, continued investigation will likely lead to the discovery of a physiologic cause for the deceleration. With quick and decisive management, these causes can be discovered and reversed, resulting in the resolution of the fetal heart rate deceleration [8]. For example, uterine tachysystole can be resolved with the discontinuation of oxytocin or the use of beta agonists such as terbutaline. Maternal hypotension from regional anesthesia placement can be resolved with the use of intravenous fluid hydration and intravenous pressors to raise the maternal blood pressure $[4,8]$.

If initial attempts at intrauterine resuscitation are unsuccessful in resolving the prolonged deceleration, the patient should be moved to the operating room and preparations should be made for emergency delivery. Guidelines for Perinatal Care by the American College of Obstetricians and Gynecologists state that patients should be monitored in the operating room until abdominal preparation has begun. If internal monitoring is being used, EFM should be continued until the completion of abdominal preparation [9]. If return to baseline is noted on arrival to the operating room, the decision to proceed with emergency cesarean delivery can be reassessed [8].

Our study compares two groups of patients, crash versus emergent cesarean sections, both of whom underwent operative delivery due to concerns for fetal well-being. The study population accounted for $13.9 \%$ and the crash cesarean group for $4.5 \%$ of all cesarean sections during the study period. The emergent group was significantly more likely to have fetal heart tones rechecked, as well as monitored on EFM while in the operating room. Foreseeably, the crash group had an overall more concerning EFM strips prior to moving the patient to the operating room with longer median prolonged deceleration and a deeper nadir of the deceleration. Despite this, there were no differences with regards to fetal outcomes. Nearly half of the crash cesarean section group who had fetal heart tones rechecked noted return to the previous fetal heart rate baseline, highlighting a group of patients for whom cesarean delivery could be potentially avoidable.

Leung et al. [14] compared 235 cases of urgent cesarean delivery for fetal bradycardia. The bradycardia-to-delivery interval and its relationship to umbilical cord $\mathrm{pH}$ values at delivery were compared in three groups based on the cause of the fetal bradycardia (irreversible, potentially reversible, and unknown groups). Overall, they did not show deterioration of the umbilical cord $\mathrm{pH}$ with time with regards to the entire study cohort (all groups). Subgroup analysis did reveal an inverse relationship between arterial $\mathrm{pH}$ and bradycardiato-delivery interval in the irreversible group. They concluded that, in cases with an unknown cause, the cord $\mathrm{pH}$ was satisfactory and there was no worsening of the $\mathrm{pH}$ with time. In our cohort, as long as irreversible causes are ruled out, taking extra time to place a patient on EFM on arrival to the operating room will not cause a significant deterioration in umbilical arterial $\mathrm{pH}$.

The crash cesarean section group was found to have a higher rate of category 1 fetal heart rate tracing (33.3\% vs. $11.3 \% p=0.003)$ prior to the prolonged fetal heart rate deceleration. Category 1 fetal heart rate tracing is characterized by normal baseline fetal heart rate (110-160 beats per min), moderate beat-to-beat variability, presence of accelerations, and lack of late decelerations $[5,6,8]$. Category 1 fetal heart rate tracing is reassuring and indicates that the fetus is not hypoxic or acidotic at that point in time $[5,6,8]$. This finding provides further evidence that this subset of patients in the crash group had a prolonged deceleration in response to a physiologic and reversible change in fetal status. The subset of patients will likely recover from the insult if appropriate interventions are chosen and adequate time is allowed for intrauterine resuscitation.

Another finding in our patient population was the significantly higher rate of general anesthesia in the crash cesarean delivery group versus the emergent cesarean group $(28.2 \%$ vs. $7.5 \% p=0.002$ ). Airway management is more challenging in pregnant patients due to 
physiologic changes to the airway from pregnancy and labor. Failed intubation is much more common in obstetric general anesthesia (1 in 224) versus non-obstetric general anesthesia (1 in 2230). Physiologic changes to the pulmonary system during pregnancy, such as diminished functional residual capacity and increased minute ventilation, can lead to rapid oxygen desaturation [17]. In an emergency situation, if the patient is not fasting, there could be significant risk of aspiration of gastric contents. By placing the patient on the fetal monitor on arrival in the operating room, even if cesarean section is not completely avoided, resolution of the fetal bradycardia could allow delay of cesarean for the administration of regional anesthesia, reducing risk to the mother. Further, the present investigation demonstrates a higher general anesthesia versus regional anesthesia technique with those mothers who have prolonged decelerations, even with return to baseline for "crash" and "emergent" patients. In fact, zero patients in the "crash" group and only 3.3\% in the "emergent" group had a spinal, reflecting that the only patients who had regional anesthesia were likely those with preexisting epidurals that were dosed rapidly with quick onset local anesthesia. Therefore, the opportunity to utilize regional anesthesia rather than general anesthesia in this setting is an important finding of this study, and would be of benefit in terms of best practice strategies for optimal anesthesia care. Based on the many known benefits of regional anesthesia in the setting of pregnant patients requiring rapid movement into an operating room with demonstrated fetal decelerations and return to baseline heart rate, anesthesiologists should consider their current algorithms and practice of rapid sequence general anesthesia technique, since regional anesthesia including spinal anesthesia techniques would likely be safe and could provide additional benefits intraoperatively.

Strengths of our study include that it was carried out on a high-risk patient population at a level 3 hospital with anesthesia, obstetrics, and neonatology staff present $24 / 7$, allowing for rapid performance of crash cesarean delivery if needed. Electronic medical records (EPIC) and archiving of fetal monitoring records (OBIX Perinatal Data System) during labor allowed for accurate data collection. A single EFM-certified physician evaluated all fetal monitoring strips for our study, which provided consistency for data collection. Weaknesses of our study included its retrospective nature and the inconsistency of collecting umbilical cord arterial blood gasses during the study period.

\section{Limitations}

There were a number of limitations with the present study. First, the study was conducted at a Level One Trauma Center in an academic medical center. Second, the obstetric physicians were part of a single group with similar practice habits; therefore, a general mix of different practices in different community setting was not utilized in the present investigation.

\section{CONCLUSIONS}

Our results indicate the practice of placing patients on fetal monitor upon arrival to the operating room prior to performing crash cesarean delivery could reduce a significant number of primary cesarean deliveries in the cohort of patients with prolonged decelerations in labor. This practice will allow additional time for intrauterine resuscitation to occur, providing additional time for resolution of the insult. If fetal heart tones have returned to baseline upon arrival in the operating room, the decision to proceed with cesarean delivery can be reconsidered. Additionally, a delay in surgical intervention would allow the time to deliver regional anesthesia, providing additional benefits to the patient and newborn. Ultimately, the clinical decision to proceed with cesarean delivery must be made taking into account many clinical factors and is the decision of the obstetrics provider. 


\section{ACKNOWLEDGEMENTS}

Funding. No funding or sponsorship was received for this study or publication of this article.

Authorship. All named authors meet the International Committee of Medical Journal Editors (ICMJE) criteria for authorship for this article, take responsibility for the integrity of the work as a whole, and have given their approval for this version to be published.

Disclosures. John A. Morgan, Miriam E. Hankins, Yuping Wang, Donna Hutchinson, Hannah L. Sams, John H. Voltz, Charles. E. McCathran and David F. Lewis have nothing to disclose. Alan D. Kaye is a member of the editorial board member for this journal.

Compliance with Ethics Guidelines. Maternal and neonatal information was obtained through linked charts in EPIC electronic medical record system. Electronic fetal monitoring strips were obtained using the OBIX Perinatal Data System. The Institutional Review Board (IRB) at LSUHSC - Shreveport, approved our study.

Data Availability. The datasets generated during and/or analyzed during the current study are available from the corresponding author on reasonable request.

Open Access. This article is licensed under a Creative Commons Attribution-NonCommercial 4.0 International License, which permits any non-commercial use, sharing, adaptation, distribution and reproduction in any medium or format, as long as you give appropriate credit to the original author(s) and the source, provide a link to the Creative Commons licence, and indicate if changes were made. The images or other third party material in this article are included in the article's Creative Commons licence, unless indicated otherwise in a credit line to the material. If material is not included in the article's Creative Commons licence and your intended use is not permitted by statutory regulation or exceeds the permitted use, you will need to obtain permission directly from the copyright holder. To view a copy of this licence, visit http://creativecommons.org/licenses/by$\mathrm{nc} / 4.0 /$.

\section{REFERENCES}

1. Safe prevention of the primary cesarean delivery. Obstetric Care Consensus No. 1. American College of Obstetricians and Gynecologists. Obstet Gynecol. 2014;123:693-711.

2. Osterman MJK, Martin JA. Trends in low risk cesarean delivery in the United States, 1990-2013. National vital statistics report; vol 63 no 6 . Hyattsvile, MD: National Center for Health Statistics. 2014.

3. Martin JA, Hamilton BE, Osterman MJK, Driscoll AK, Matthews TJ. Births: final data for 2015. Natl Vital Stat Report. 2017;66(1):1.

4. Chen HY, Chauhan SP, Ananth CV, Vintzileos AM, Abuhamad AZ. Electronic fetal heart rate monitoring and its relationship to neonatal and infant mortality in the United States. Am J Obstet Gynecol. 2011;204(6):491.e1-10.

5. Intrapartum Fetal Heart Rate Monitoring: Nomenclature, Interpretation, and General Management Principles. Practice Bulletin No. 106. American College of Obstetricians and Gynecologists. Obstet and Gynecol 2009; 192-202.

6. Management of intrapartum fetal heart rate tracings. Practice Bulletin No 116. American College of Obstetricians and Gynecologists. Obstet Gynecol 2010; 116: 1232-40.

7. Low JA, Victory R, Derrick EJ. Predictive value of electronic fetal monitoring for intrapartum fetal asphyxia with metabolic acidosis. Obstet Gynecol. 1999;93:285-91.

8. Freeman, Roger KMD. Fetal heart rate monitoring. 4th ed. Philadelphia: Lippincott Williams \& Wilkins; 2012.

9. American Academy of Pediatrics \& American College of Obstetricians and Gynecologists. Guidelines for perinatal care (7th edition). Washington, DC: Elk Grove; 2012. p. 192-4.

10. Lagrew DC, Bush MC, McKeown AM, Lagrew NG. Emergent (crash) cesarean delivery: indications and 
outcomes. Am J Obstet Gynecol. 2006;194: 1638-43.

11. Nageotte MP, Vander Wal B. Achievement of the 30-minute standard in obstetrics-can it be done? Am J Obstet Gynecol Patient Saf Ser. 2012;206(2): 104-7.

12. Boehm FH. Decision to incision: time to reconsider. Am J Obstet Gynecol. 2012;206(2):97-8.

13. Williams KP, Galerneau F. Fetal heart rate parameters predictive of neonatal outcome in the presence of a prolonged deceleration. Obstet Gynecol. 2002;100:951-4.
14. Leung TY, Chung PW, Rogers MS, Sahota DS, Lao TT, Chung TK. Urgent cesarean delivery for fetal bradycardia. Obstet Gynecol. 2009;114:1023-8.

15. Wu RW, Chen CP, Wang KG. Implications of prolonged fetal heart rate deceleration during the second stage of labor. J Formos Med Assoc. 1996;95: 231-5.

16. Cahill AG, Caughey AB, Roehl KA, Odibo AO, Macones GA. Terminal fetal heart decelerations and neonatal outcomes. Obstet Gynecol. 2013;122: 1070-6.

17. Obstetric analgesia and anesthesia. Practice Bulletin No. 177. American College of Obstetricians and Gynecologists. Obstet Gynecol 2017; 129:e73-89. 